O ne of the former $E B M H$ editors, Peter Szatmari, left us with a parting request: that we should try to include six abstracts relating to child and adolescent mental health in every issue. This, he argued, was only fair given that kids make up roughly one quarter of the population. That we manage to achieve this fairly consistently is an indicator of the vitality of this area of research. A glance at the headlines in the medical and lay press indicates why this should come as no surprise: the rising prevalence of autistic spectrum disorders and possible explanations; ADHD and the role of psychostimulants; SSRIs and depression in adolescents. Weighty issues with relevance to all mental health professionals, regardless of specialty, as we know that childhood adversity and psychopathology has a profound impact on adult lives. In this issue of the journal, we have two special articles focusing on children and adolescents. The Department of Health for England has recently revised its guidance on alcohol consumption in pregnancy, now advocating complete abstinence rather than the occasional drink. Kapil Sayal (page 98) looks at what we really know about the effects on mental health of prenatal exposure to alcohol. Despite a flurry of recent trials and guidelines, it is still not clear how best to treat depression in adolescents. This is partly due to the shadow cast over the prescription of SSRIs following the selective reporting of adverse effects-notably suicidal thinking -in drug trials. Also in this issue, Bernadka Dubicka and Paul Wilkinson sort through the available evidence and find that the UK NICE guidance is already out of date (page 100).

Editor, Evidence-Based Mental Health

\title{
Alcohol consumption in pregnancy as a risk factor for later mental health problems
}

T here has been increasing interest in recent years about the possible mental health risks for offspring as a result of prenatal alcohol exposure. In particular, there is considerable controversy about whether there is a safe threshold for alcohol consumption during pregnancy and whether international policy recommendations are based on evidence. This article briefly summarises the existing literature in relation to mental health outcomes in childhood, adolescence and early adulthood following prenatal alcohol exposure. It also highlights some of the possible pitfalls in the interpretation of observational epidemiological data.

There are currently considerable international differences in policy recommendations about alcohol use in pregnancy. Some countries such as the USA, Canada and France have recommended complete abstinence. This appears to be based on a precautionary approach, given that no clear safe threshold for drinking has been established. In contrast, until May 2007, guidelines in the UK were that pregnant women could safely drink one or two drinks per occasion up to 1-2 times per week (that is, up to a total of four drinks/ units of alcohol per week). This approach appears to be based on the lack of available evidence demonstrating that low levels of alcohol consumption are associated with later adverse outcomes. These recommendations were recently revised to advise abstinence. However, this revision was based on the perceived greater clarity of a "no drinking" message rather than on the research evidence, and the guidelines remain ambiguous as they state that women who choose to drink can drink up to the previous limits. ${ }^{1}$

\section{LOW TO MODERATE ALCOHOL CONSUMPTION}

A recent systematic review carried out by the National Perinatal Epidemiology Unit focused on the literature relating to risks associated with low to moderate levels of alcohol consumption and binge drinking during pregnancy. ${ }^{2}$ There is more conclusive evidence related to higher levels of drinking, manifesting at the extreme end as fetal alcohol syndrome. In the USA, there has been acknowledgment of partial forms of these outcomes, and the prevalence of fetal alcohol spectrum disorder (FASD) had been estimated at around $1 \%$ of live births. ${ }^{3}$ More recently, in Europe, rates of FASD have been estimated at $2-4 \%{ }^{4}$ The majority of these children are described as having an alcohol-related neurodevelopmental disorder (ARND), manifesting in terms of problems with overactivity, inattention, behaviour or learning. These outcomes have been found to be associated with moderate levels of exposure (for example, an average of one drink per day) and these findings have been replicated in animal studies, suggesting that there may be critical vulnerable periods for neurodevelopment. There is greater controversy about the possible harmful effects of lower levels of drinking (ranging between occasional drinking and less than one drink per day during pregnancy). There has been limited published research investigating the role of low levels of exposure, which may reflect a publication bias if there have been mainly negative findings. Often where these findings have been presented in papers, the role of lower levels of alcohol use has been investigated as part of a wider range that includes higher levels of drinking. Therefore, findings involving lower levels have not usually been adjusted 
specifically for key confounding variables. However, at least two studies suggest that low levels of drinking during pregnancy may be associated with childhood mental health problems. A study in the USA found that up to 3-4 drinks per week was associated with behavioural problems at ages 6-7 years, after adjusting for confounders. ${ }^{5}$ These findings were recently extended by Sayal et al in the UK who found that less than one drink per week in the first trimester was associated with higher levels of mental health problems between the ages of $4-8$ years as assessed by both parents and teachers. ${ }^{6}$ This association was only demonstrated in girls, highlighting the need for further replication of these findings.

The consumption of moderate levels (1-2 drinks per day) of alcohol during pregnancy has been found to be associated with childhood attentional and behavioural problems. ${ }^{7-11}$ There is increasing evidence that the risks from alcohol consumption in pregnancy may persist over time. Cohort studies that have reported data collected during adolescence and early adulthood have described ongoing learning problems, behavioural difficulties, and adult psychiatric disorders such as personality and substance use disorders. ${ }^{12-}$ ${ }^{14}$ However, these findings need to be interpreted cautiously in terms of the potential caveats outlined below.

\section{METHODOLOGICAL ISSUES}

Observational epidemiological research investigating the aetiological role of environmental factors during pregnancy in contributing to later psychopathology is beset by methodological difficulties. The key methodological concerns relating to bias and confounding will be described. First, bias can be considered in terms of selection and information bias. Case-control studies are particularly susceptible to selection bias because of their frequent reliance on clinical samples to identify the cases. These are subject to referral bias and may provide misleading estimates of the risk from the exposure. Ideally, to demonstrate causal associations involving prenatal alcohol exposure, prospective longitudinal studies involving large sample sizes which are representative of the wider population are required. However, there are inherent difficulties with this in longitudinal research. Recruitment approaches adopted by investigators (such as the levels of generalisability from the choice of baseline sample) and high levels of long-term loss to follow-up mean that findings need to be interpreted with caution. Sample attrition over time may also be systematically associated with key exposure, confounder and outcome variables meaning that findings are only partially generalisable. International differences and changes in temporal trends in drinking during pregnancy also mean that findings need to be interpreted cautiously. Pregnant women who consumed alcohol at a particular timepoint (such as during a particular decade) and in a particular culture may not be generalisable to a sample from a different time and place. For example, the correlates of women who drank heavily during pregnancy in the 1970s may be very different from those who drink heavily now, given the greater knowledge of the possible risks.

With regard to information bias, both exposure and outcome status are vulnerable to measurement errors. In particular, there may be recall bias with regard to ascertainment of the exposure status (especially if enquiries are made postnatally). Prospective research designs are required that ascertain the timing, frequency, amount, duration, pattern and variability of drinking across the course of the pregnancy. Even then, several studies suggest that an important risk period is before pregnancy recognition, ${ }^{12}{ }^{15}$ a period that is particularly vulnerable to recall bias about the exposure.
When relying on self-report, there is considerable risk of underestimating alcohol consumption. This can result in systematic misclassification with women who drink heavily reporting their consumption as moderate, and those who drink moderately reporting light or no consumption, thus contributing to misleading risk estimates. There may also be a tendency to provide socially desirable responses. Studies comparing levels of alcohol consumption elicited through concurrent and retrospective self-report have found that higher levels are reported retrospectively. ${ }^{16}$ Despite all these factors, self-reports of alcohol consumption have been shown to be valid in relation to predicting risk for neurobehavioural outcomes. ${ }^{11}$ Methodological approaches that average out weekly amounts of drinking might mask binge episodes of alcohol consumption and fetal exposure to peak levels of alcohol. Similarly, the averaging out of levels over longer time periods might obscure risks related to particular timing of the exposure. Finally, outcome measures should ideally involve a range of informants during childhood (for example, parents and teachers) or involve reliable and valid diagnostic interview measures.

Second, there are a range of alternative factors that might explain an apparent relation between alcohol consumption in pregnancy and later mental health difficulties. Prenatal confounding variables including the use of other substances, smoking, background socioeconomic factors, maternal anxiety and depression, and maternal nutrition, might be associated with both the exposure and the outcome. As above, the measurement of confounders (in particular, the use of other substances and smoking during pregnancy) should also be rigorous to minimise information bias. Poorly measured or unmeasured confounders may otherwise account for a spurious association between a risk factor and an outcome. Even if analyses control for a large number of confounding variables, residual confounding remains a possibility. The postnatal environment, particularly involving parental mental health or quality of parenting, may include more proximal mechanisms through which the apparent risk is transmitted. Genetic predisposition, on both the maternal and paternal side, needs to be taken into account when considering how the risk might be transmitted. For example, an apparent association between prenatal alcohol exposure and childhood psychopathology disappeared when controlling for family risk for alcohol dependence. ${ }^{17}$ There are also likely to be gene-environment correlations whereby the propensity to drink during pregnancy may be closely related to the genetic risk of transmission of mental health disorders. Finally, given that not all children who have been exposed to high levels of alcohol consumption in pregnancy have adverse outcomes, there may be gene-environment interactions whereby a combination of risk factors is required.

\section{CONCLUSIONS}

There is a challenge for future research designs to account for the potential range of prenatal environmental risk factors for later mental health problems and how they might operate in combination with genetic risk. In the meantime, there is also a need for clarity from policy makers in terms of key public health and health education messages for pregnant women and women of child-bearing age who might be contemplating becoming pregnant. As yet, a safe threshold has not been conclusively established and it is important that accurate information about possible risks is available so that women can make informed decisions.

KAPIL SAYAL Section of Developmental Psychiatry, University of Nottingham, Nottingham NG7 2UH, UK

Competing interests: None. 


\section{REFERENCES}

1 Department of Health. The Pregnancy Book, 2007. UK: DH, 2007, Available at http://www.dh.gov.uk/en/Publicationsandstatistics/Publications/ PublicationsPolicyAndGuidance/DH_074920 (accessed 19 September 2007).

2 Gray R, Henderson J. Review of the fetal effects of prenatal alcohol exposure: report to the Department of Health, National Perinatal Epidemiology Unit, University of Oxford, 2006

3 Sampson PD, Streissguth AP, Bookstein FL, et al. Incidence of fetal alcohol syndrome and prevalence of alcohol-related neurodevelopmental disorder. Teratology 1997;56:317-26.

4 May PA, Fiorentino D, Gossage PJ, et al. Epidemiology of FASD in a province in Italy: Prevalence and characteristics of children in a random sample of schools. Alcohol Clin Exp Res 2006;30:1562-75.

5 Sood B, Delaney-Black V, Covington C, et al. Prenatal alcohol exposure and childhood behavior at age 6 to 7 years: I. dose-response effect. Pediatrics 2001;108:E34

6 Sayal K, Heron J, Golding J, et al. Prenatal alcohol exposure and gender differences in childhood mental health problems: a longitudinal populationbased study. Pediatrics 2007;119:e426-34.

7 Brown RT, Coles CD, Smith IE, et al. Effects of prenatal alcohol exposure at school age, Il: attention and behavior. Neurotoxicol Teratol 1991:13:369-76.

8 Burden MJ, Jacobson SW, Sokol RJ, et al. Effects of prenatal alcohol exposure on attention and working memory at 7.5 years of age. Alcohol Clin Exp Res 2005;29:443-52.

9 Mick E, Biederman J, Faraone SV, et al. Case-control study of attention-deficit hyperactivity disorder and maternal smoking, alcohol use, and drug use during pregnancy. J Am Acad Child Adolesc Psychiatry 2002:41:378-85.

10 Nordstrom Bailey B, Delaney-Black V, Covington CY, et al. Prenatal exposure to binge drinking and cognitive and behavioral outcomes at age 7 years. Am J Obstet Gynecol 2004;191:1037-43.

11 Jacobson SW, Chiodo LM, Sokol RJ, et al. Validity of maternal report of prenatal alcohol, cocaine, and smoking in relation to neurobehavioral outcome. Pediatrics 2002;109:815-25.

12 Carmichael Olson H, Streissguth AP, Sampson PD, et al. Association of prenatal alcohol exposure with behavioral and learning problems in early adolescence. J Am Acad Child Adolesc Psychiatry 1997;36:1187-94.

13 Alati R, Al Mamun A, Williams GM, et al. In utero alcohol exposure and prediction of alcohol disorders in early adulthood: a birth cohort study. Arch Gen Psychiatry 2006;63:1009-16.

14 Barr HM, Bookstein FL, O'Malley KD, et al. Binge drinking during pregnancy as a predictor of psychiatric disorders on the structured clinical interview for DSM-IV in young adult offspring. Am J Psychiatry 2006;163:1061-5.

15 Streissguth AP, Barr HM, Sampson PD. Moderate prenatal alcohol exposure: effects on child IQ and learning problems at age 7 1/2 years. Alcohol Clin Exp Res 1990;14:662-9.

16 Alvik A, Haldorsen T, Groholt B, et al. Alcohol consumption before and during pregnancy comparing concurrent and retrospective reports. Alcohol Clin Exp Res 2006;30:510-15.

17 Hill SY, Lowers L, Locke-Wellman J, et al. Maternal smoking and drinking during pregnancy and the risk for child and adolescent psychiatric disorders. J Stud Alcohol 2000;61:661-8.

\section{Evidence-based treatment of adolescent major depression}

A dolescent depression is a serious and debilitating disorder (see box). Up to one in 20 adolescents suffer from major depression at any point in time, and $20 \%$ of adolescents have at least one episode of clinical depression by the age of 18. Once depression is established it often becomes chronic. About a fifth of adolescents with major depression will continue to have a persistent disorder, and another third will recover but go on to have recurrent episodes.

\section{CAUSATION}

Depression runs in families and children of depressed parents have an increased risk of becoming depressed, which is likely to be due to both the direct effect of genes as well as the adverse influence of depressed parenting. Recent research has highlighted the complex interaction of genetic and environmental influences, such as adverse life events, in depression.

\section{COMPLICATIONS}

Pure depression is rare, and it is usually accompanied by other psychiatric disorders. A recent UK study found that $89 \%$ of cases had a comorbid disorder and the average number of additional disorders was three. ${ }^{1}$ Other complications include school refusal, academic failure, impaired peer relations, drug and alcohol abuse, and family relationship problems. However, the most important complication is suicide and depression is the most important risk factor for

\section{The six Cs of depression}

- Common

- Chronic

- Causation complex

- Comorbidity

- Complications

- Commit suicide suicidality. Findings from 20-year follow-up data of depressed children and adolescents have shown that $2.5 \%$ had committed suicide and nearly half had attempted suicide.

Any treatment plan for adolescent depression therefore needs to take account of the chronic, relapsing nature of the disorder, consider aetiological factors such as parental depression, and address any concurrent psychiatric disorder and psychosocial complications, particularly suicidality.

\section{EVIDENCE-BASED PSYCHOLOGICAL TREATMENT}

Early studies of cognitive behavioural therapy (CBT) were promising in the prevention and treatment of depression and meta-analyses found large effect sizes. However, a recent meta-analysis, which included studies with active controls, reported a relatively small effect size of $0.3 .^{2}$ The largest study, the Treatment of Adolescent Depression Study (TADS), which had more severely impaired participants than earlier studies, found that CBT alone did not differ from placebo on any of 15 outcomes. ${ }^{3}$ However, there is evidence from a sub-analysis that CBT may be effective in adolescents from higher income families ${ }^{4}$ and for high levels of cognitive disorders.

Recent findings in adult depression present an interesting line of evidence for the treatment of more severe depressive disorder. Dimidjian and colleagues reported that behavioural activation (using activity scheduling and graded tasks) without a cognitive component was comparable to antidepressant medication, and both significantly outperformed cognitive therapy. ${ }^{5}$ These results suggest that behavioural activation may be a useful treatment for more severe disorders and a viable alternative to antidepressant medication, although this needs to be tested in adolescents.

There have been three randomised controlled trials (RCTs) with positive results for interpersonal therapy (IPT). One trial was particularly encouraging as IPT was delivered by social workers with only basic training in therapeutic skills and proved to be superior to treatment as usual from school mental health teams. ${ }^{6}$ However, there are very few trained IPT therapists in the UK. 